\title{
Investigating time and economic costs of botrytis bunch rot sampling using interpolated data
}

\author{
Gareth N. Hill ${ }^{*}$, Peter Jaksons ${ }^{2}$, Joanna M. Sharp ${ }^{2}$, Adrian G. Hunt ${ }^{3}$ and Kai S.J. Lewis ${ }^{1}$ \\ ${ }^{1}$ The New Zealand Institute of Plant and Food Research, Private Bag 92169, Auckland Mail \\ Centre, Auckland 1142, New Zealand \\ ${ }^{2}$ The New Zealand Institute of Plant and Food Research, Private Bag 4704, Christchurch Mail \\ Centre, Christchurch 8140, New Zealand \\ ${ }^{3}$ The New Zealand Institute of Plant and Food Research, Private Bag 1401, Havelock North \\ 4157, New Zealand \\ *Corresponding author: gareth.hill@plantandfood.co.nz
}

\begin{abstract}
Botrytis cinerea causes botrytis bunch rot (BBR) disease in wine grapes. Small-scale labour-intensive visual disease assessments may not adequately represent an entire vineyard but larger assessments add cost without necessarily improving accuracy or financial returns. BBR-severity data were collected on three dates from two sites and spatially interpolated. Balanced acceptance sampling (BAS) and simple random sampling (SRS) were compared using sample sizes of 2 to 200 vines. Assessment times were calculated for both walking (rows ignored) and driving (rows impassable) and costs compared with assessment error and effects on crop value. Overall, BAS performed better than SRS. Driving was faster than walking except when sample distribution necessitated travelling down every row regardless of sample size. Annual crop losses of up to NZ\$2578 per hectare could result from short assessment times and subsequent inaccurate estimates of BBR severity. Spatial interpolation was shown to be a useful and promising technique for studying BBR sampling strategies in vineyard blocks. Travel was not a substantial component of assessment time. An 80-minutelong assessment could substantially reduce economic losses because of errors in BBR assessments.
\end{abstract}

Keywords BBR, grey mould, grapevines, spatial analysis, interpolation, sampling strategies, balanced acceptance sampling, simple random sampling.

\section{INTRODUCTION}

Botrytis bunch rot (BBR), caused by Botrytis cinerea, is one of the most economically important diseases of wine grapes (Hill et al. 2010) and is known to have substantial spatial variability in vineyards (Bramley et al. 2011a; McKay et al. 2012). McKay et al. (2012) used artificially generated datasets to compare three BBR sampling strategies: simple random sampling (SRS), inverse sampling (sampling continues until a predefined number of diseased bunches is recorded), and adaptive cluster sampling (sampling intensity increases when disease bunches are found in the vicinity of infected bunches). They found that optimal sampling procedures depend on distribution of BBR within a vineyard block. Bramley et al. (2011a) studied the spatial variability of BBR in a vineyard, but not for the purpose of optimising sampling.

Vineyard sampling, regardless of the characteristic or variable being measured, is a difficult thing to do well, as accuracy and precision must be weighed up against the cost of sampling, 
both in terms of time and financial costs. The average New Zealand vineyard area is 18.5 ha (New Zealand Winegrowers 2018), providing the potential for variability across a wide area, so optimising sampling strategies is critical. Despite this, Reynolds and de Savigny (2004) note that it is common for wineries to conduct minimal sampling, with one large winery only sampling 10 random bunches per vineyard block, unless it is "large", in which case they collect two 10-bunch samples. This sampling strategy is unlikely to account for the variability inherent in over 80,000 grape bunches per hectare (Reynolds et al. 2004). Without properly understanding variability in the vineyard, whether that be yield, fruit quality or disease, it is not possible to adequately plan management interventions or harvest logistics.

Insufficient sampling is not a symptom of a lack of research into optimal sampling strategies, as many studies have addressed this issue for particular characteristics. Taylor and Bates (2012) found that around 64 vines were needed to estimate mean pruning weights with acceptable error (defined as $\pm 0.046 \mathrm{~kg} / \mathrm{m}$ ) and 90\% confidence. Kasimatis and Vilas (1985) studied sampling strategies for soluble sugar content and found that any sampling that focused on berry sampling rather than cluster sampling did not reliably represent vineyard maturity. Sampling studies have also been conducted for a range of insect pests, including mealybugs (Geiger \& Daane 2001), European grapevine moth (Ifoulis \& Savopoulou-Soultani 2006), and ant populations (Mgocheki \& Addison 2009), and diseases, including Downy mildew (Seem et al. 1985), Pierce's disease (Park et al. 2006; Park et al. 2011), and leafroll virus (Arnold et al. 2016).

The vast majority of these studies focus on sampling-strategy optimisation of sample size and overall error compared with population means. However, there are other aspects to consider regarding optimal sampling strategies, namely spatial variation and the costs of assessment, both in terms of time and economics. As McKay et al. (2012) found, understanding spatial variation is crucial to optimising sampling, and the emerging field of precision viticulture has the goal of achieving that (Bramley 2010). Various studies have used precision viticulture practices and spatial analysis to better understand aspects of vineyard variability, such as physical vine characteristics (Bramley et al. 2011b) and fruit composition and juice quality (Trought \& Bramley 2011). Bramley et al. (2011a) also used these approaches to study both powdery mildew and BBR.

Once variability is understood at the vineyard scale, sampling strategies can be set that directly target that variability in order to collect the best estimate of population means with minimal effort. Meyers and Vanden Heuvel (2014) used aerial multispectral imagery to calculate the normalised difference vegetation index (NDVI) of a vineyard to use as a relatively easily gathered proxy for overall variability in order to select areas of the vineyard that best represent the vineyard as a whole. This approach allows the sampling strategy to be set per vineyard and at the time of sampling, although it relies on the assumption that NDVI variability is a reasonable proxy for the characteristic being sampled for. González-Fernández et al. (2019) used spatially interpolated vineyard maps and a fuzzy K-means algorithm (a common unsupervised machinelearning method) to establish unique vineyard management zones based on their variability. This takes targeted sampling one step further by identifying areas of a vineyard that are unique in their variability and, therefore, may need different sampling strategies.

The present study aimed to understand the costs of BBR assessments, both in terms of time and economic costs, as well as the overall error. High-spatial resolution BBR datasets were generated through the mapping of BBR in three vineyard blocks and spatially interpolating disease severity across the entire blocks. Sampling strategies were then compared in terms of error as well as the time and economic costs. The time component of the analysis compared undertaking both assessments on foot, where assessors can move between vine rows, and assessment using farm vehicles, where assessors can only move within rows. The economic component of the 
analysis involved estimating the labour costs based on assessment time, as well as virtual crop losses due to inaccurate assessments affecting the amount paid for the crop.

\section{MATERIALS AND METHODS}

\section{Disease assessments}

Botrytis bunch rot (BBR) disease assessments were carried out in two Sauvignon blanc vineyards in Hawke's Bay, New Zealand: one on Gimblett Rd $(-39.608,176.740)$ and one on Korokipo Rd $(-39.580,176.767)$. Two assessments were carried out at Gimblett Rd in different blocks of vines, the first on 1 March 2018 (GimblettRd1, 0.32 ha; longest length $=34.5 \mathrm{~m}$; longest width $=15.6 \mathrm{~m}$ ) and the second on 08 March 2018 (GimblettRd2, 0.33 ha; longest length $=288.0 \mathrm{~m}$; longest width $=16.1 \mathrm{~m}$ ). A single assessment was carried out in a block of vines at Korokipo Rd on 7 March 2018 (KorokipoRd; 0.35 ha; longest length $=78.1 \mathrm{~m}$; longest width $=52.9 \mathrm{~m}$ ). The position of each assessed vine was recorded by placing a Geo 7X real-time kinetic (RTK) GPS system (Trimble, Sunnyvale, CA, USA) at the base of the vine and allowing the device to record at least 30 points (one point per second) and take an average. Sets of three to five neighbouring vines were assessed at irregular intervals along each vine row assessed, with every second row being assessed within each block (Fig. 1). The total number of vines assessed was dependent on the amount of time available to perform the assessments and, therefore, varied between blocks. Disease assessments were carried out using the image-analysis approach outlined by Hill et al. (2017), whereby grape bunches are photographed against a blue background (cotton pillow case) and the images later cropped to the bunch and processed using RotBot software (Hill et al. 2014).

Bunches were assigned to vines by matching the timestamps on the photos with the timestamp of each recorded GPS point. Multiple bunches were photographed within each vine while the GPS points were being recorded and averaged to a single point for that vine; therefore it was not possible to easily assign a unique GPS point to each photograph/bunch. Consequently, GPS coordinates were assigned to bunches if the timestamp on the photograph was greater than the previous GPS point and less than the following GPS point.

\section{General data analysis and mapping}

All general data analysis and mapping were carried out using R software (v3.5.0; R Core Team 2018), with the exception of block dimensions which were calculated using QGIS (v3.6.0). The tidyverse (v1.2.1; Wickham 2017) and ggplot2 (v3.1.0; Wickham 2016) packages were used for data manipulation and plotting. The ggmap
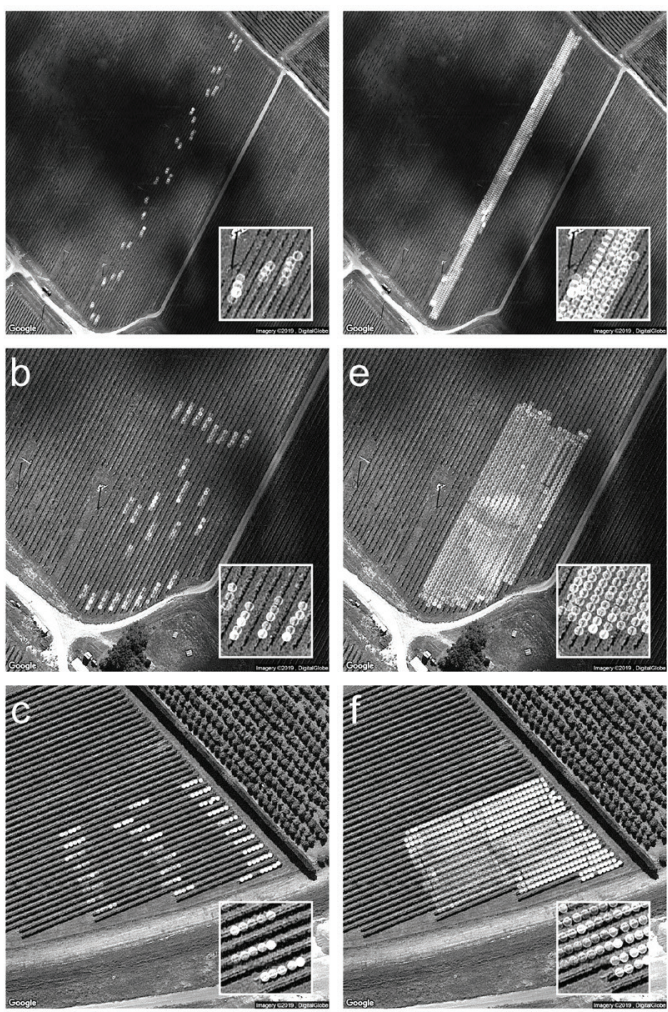

BBR severity (\%)

Figure 1 Actual $(a, b, c)$ and interpolated $(d, e, f)$ botrytis bunch rot (BBR) severity at GimblettRd1 (a, d), GimblettRd2 (b, e) and KorokipoRd (c, f). Insets show a zoomed arbitrary region of each map. 
v3.0.0; Kahle \& Wickham 2013) package was used to generate maps.

\section{Interpolation}

Interpolation was carried out in $\mathrm{R}$ using the geosphere (v1.5-7; Hijmans 2017) and interp (v1.0-31; Gebhardt et al. 2018) packages. Both vines and $\mathrm{BBR}$ were interpolated using piecewise linear interpolation (Franke 1979), which is the default method used by the interp package.

The positions of non-assessed vines were interpolated by finding gaps in assessed rows and filling them with interpolated vines based on geospatial characteristics of each assessed vine row. The first and last vine in each row were determined and vines were ranked within each row by the distance from the first vine in that row, thus reordering vines by their position in the row in case they were not assessed in sequence. Vine spacing was calculated for each row as the median distance between vines if less than $3 \mathrm{~m}$, which excluded gaps of non-assessed vines from the median calculation. The overall bearing of each assessed row was then calculated using each vine in sequence moving from the first to last vine. Non-assessed vine positions could then be interpolated by iterating through the vines in a row, identifying gaps between assessed vines of more than 1.5 times the median vine spacing and using the row bearing to generate a new vine location at intervals equal to the median vine spacing in line with the rest of the vine row.

Entire vine rows in between assessed vine rows were also interpolated to provide a full set of vine positions within each block. Each assessed and interpolated vine in assessed rows was duplicated $90^{\circ}$ from the row bearing at a distance of $2.5 \mathrm{~m}$ (pre-established row spacing) in order to simulate an entire block.

Mean BBR was calculated for each assessed vine and interpolated across each vineyard block for all interpolated vines. The error in the interpolation method was investigated for each block using 100 random cross-validations. For each of these cross-validations, $20 \%$ of the assessed vines were assigned to the validation set, the remaining $80 \%$ was used to interpolate both non-assessed vines and BBR, and then the interpolated $\mathrm{BBR}$ was correlated against actual BBR.

\section{Simulated sampling strategies}

Sampling simulations and assessment distance calculations were carried out in R using the SDraw (v2.1.3; McDonald 2016), sp (v1.3-1; Pebesma \& Bivand 2005) and TSP (v1.1-6; Hahsler \& Hornik 2018) packages.

Samples of various sizes (number of vines) were simulated using two sampling strategies: Simple Random Sampling (SRS; Cochran 1977), which is spatially random, and Balanced Acceptance Sampling (BAS; Robertson et al. 2013), a spatially balanced sampling design, which has an underlying algorithm that spreads sampling points evenly over space. Monte Carlo simulations (Gentle et al. 2012) were run for each vineyard block with all sample sizes of between 2 and 200 vines. These simulations were run 100 times for each sample size for each sampling strategy (SRS and BAS). This resulted in a total of 119,400 subsamples of vines across all three blocks.

The arbitrary insertion algorithm was used to estimate the walking travel time for each sample via the fastest route between the sampling points. This algorithm is commonly implemented to optimise the 'travelling salesman problem' (TSP; Sathya \& Muthukumaravel 2015). This method assumes that is possible to move freely between rows at any given point by ducking under vines. Walking pace was assumed to be $5 \mathrm{~km} / \mathrm{h}$ for assessment time calculations.

To estimate the travel time using a motorised vehicle such as a quad bike, it was assumed that it is only possible to move between rows at the beginning or end of a row. To calculate the travel time within each row, the distance from the beginning or end of the row, whichever was the least, to the sampled vine(s) was measured. If it was faster to reverse out of a given row the distance was multiplied by two, otherwise it was assumed that the total travel distance would be the length of the row. An additional $2.5 \mathrm{~m}$ of travel distance between each consecutive row 
was added. Vehicle speed was assumed to be 30 $\mathrm{km} / \mathrm{h}$ for assessment time calculations.

Time for assessing vines was added to all simulations in order to calculate overall assessment time, and was assumed to be $1 \mathrm{~min}$ per vine for the purposes of these calculations. Crop loss per hectare was calculated by multiplying the absolute error in BBR for each sample by the mean price per hectare of grapes in New Zealand in 2017, which was $\$ 18,746.40$ (New Zealand Winegrowers 2018). This was based on the premise that grapes with BBR have no monetary value. An analysis that included the economic cost of assessments was considered; however, this would have been equivalent to the time cost multiplied by a pre-defined hourly rate, and therefore would not have added any additional information.

\section{RESULTS}

\section{Disease assessments and interpolation}

The number of bunches assessed per vine varied both within and between vineyard blocks, as did mean BBR severity (Table 1). The comparative area of each block was similar, although number of vines assessed and interpolated varied because of vine spacing at each site, with a greater proportion of vines being interpolated at
GimblettRd1 (Table 2).

Severity of BBR was interpolated and mapped at all three vineyard blocks (Fig. 1). Crossvalidation showed that interpolated $\mathrm{BBR}$ was not correlated with actual BBR at GimblettRd1 $(\mathrm{r}=0.039)$, weakly correlated at GimblettRd2 $(\mathrm{r}$ $=0.309)$, and well correlated at KorokipoRd $(\mathrm{r}=$ 0.833).

\section{Sampling strategy comparison}

Samples taken using the balanced acceptance sampling (BAS) strategy resulted in mean BBR values that appeared to converge to the actual mean BBR faster than samples taken using the simple random sampling (SRS) approach (Fig. 2). Variance between samples of similar sizes for both sampling strategies decreased as sample size increased and remained relatively stable with sample sizes greater than 100 vines. Variance resulting from BAS was higher for some samples of very few vines $(n<5)$, but was lower than SRS for all other sample sizes.

\section{Travel and assessment time}

Travel time for vehicle-based sampling using a row-by-row sampling strategy was shorter than sampling by walking ignoring vine rows (Fig. 3). There was a minimum travel time when

Table 1 Number of bunches assessed for botrytis bunch rot (BBR) per vine and mean BBR for each vineyard block. Standard error shown in parentheses.

\begin{tabular}{lllc}
\hline Vineyard block & Bunches assessed (range) & Bunches assessed (mean) & Mean BBR (\%) \\
\hline GimblettRd1 & $1-15$ & $7.8( \pm 0.1)$ & 1.9 \\
GimblettRd2 & $2-17$ & $9.6( \pm 0.1)$ & 4.9 \\
KorokipoRd & $4-18$ & $9.5( \pm 0.1)$ & 13.9 \\
\hline
\end{tabular}

Table 2 Numbers of assessed and interpolated vines for each vineyard block.

\begin{tabular}{llllll}
\hline $\begin{array}{l}\text { Vineyard } \\
\text { block }\end{array}$ & $\begin{array}{l}\text { Assessed } \\
\text { vines }\end{array}$ & $\begin{array}{l}\text { Interpolated } \\
\text { vines }\end{array}$ & $\begin{array}{l}\text { Total } \\
\text { vines }\end{array}$ & $\begin{array}{l}\text { Area } \\
(\text { ha })\end{array}$ & $\begin{array}{l}\text { Vine spacing } \\
(\mathrm{m})\end{array}$ \\
\hline GimblettRd1 & 98 & 750 & 848 & 0.32 & 1.85 \\
GimblettRd2 & 122 & 658 & 780 & 0.33 & 1.82 \\
KorokipoRd & 127 & 523 & 650 & 0.35 & 1.99 \\
\hline
\end{tabular}




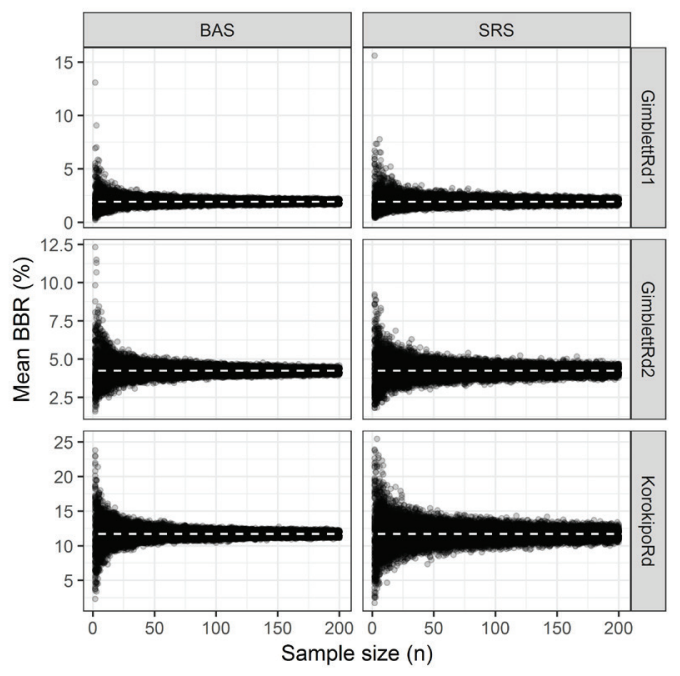

Figure 2 Comparison of mean botrytis bunch rot (BBR) balanced acceptance sampling (BAS) and simple random sampling (SRS) for sample sizes of 2-200 vines at all three vineyard blocks. Dashed white line indicates actual mean BBR at each block.

driving for some samples regardless of sample size, which can be seen in the points at the far right of the $x$-axis in Figure 3. This effect was more apparent at GimblettRd1 and GimblettRd2 because of a distinct vertical line of points, but can also be seen as a sharp cut-off at KorokipoRd. The maximum travel time for samples of 200 vines was $2.84 \mathrm{~min}$ when driving and $11.05 \mathrm{~min}$ when walking, excluding the time required for assessing BBR on each vine.

Assessment time per vine was assumed to be $1 \mathrm{~min}$, and therefore the total time required for assessments was simply travel time plus sample size.

\section{Economics of sampling}

Additional crop loss in NZ\$ per hectare resulting from errors in BBR assessments varied considerably with sample size because of variation in sample error. However, this loss generally reduced with sample size and was consistently below NZ\$500 per hectare with assessment times of greater than $80 \mathrm{~min}$ (Fig. 4). The median crop

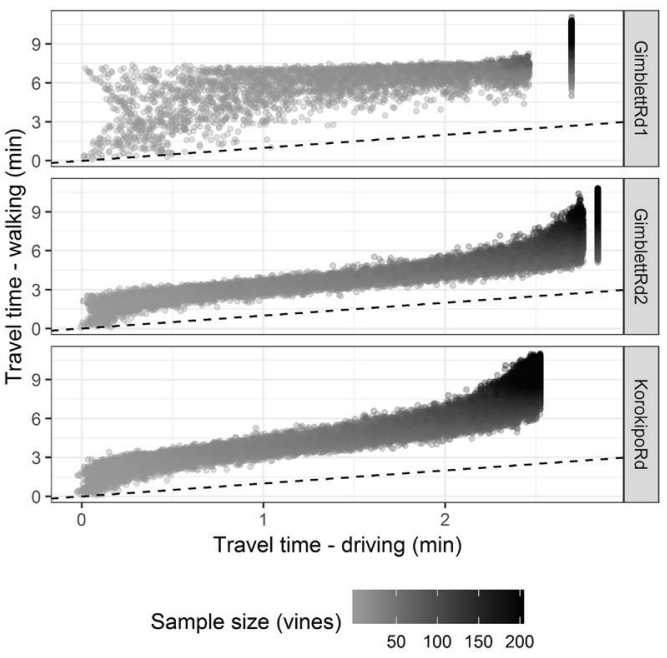

Figure 3 Comparison of travel time at all three vineyard blocks when walking $(5 \mathrm{~km} / \mathrm{h}$; can travel between rows) or driving $(30 \mathrm{~km} / \mathrm{h}$; can only travel within rows). Dashed line represents 1:1 correlation between walking and vehicle travel times.

loss was $\$ 36.14$ per hectare, while the maximum crop loss was $\$ 2577.67$ per hectare, based on 2017 figures. The random sample this maximum crop loss amount was calculated for had a sample size of four vines and an absolute error of $13.75 \%$.

\section{DISCUSSION}

Interpolating entire vineyard blocks from smaller samples of that block is one way of optimising sampling strategies in order to obtain a better understanding spatial variability with reduced sampling. This interpolation sampling approach worked well in this study, although there were some issues with the methodology. Inconsistent data on the numbers of bunches per vine may have been partly because of availability of bunches on the vine due either to yield variation or inconsistent sampling. However, it was more likely because of errors in the attribution of bunches to vines based on misaligned timestamps of photos taken for image analysis and recorded GPS locations. Using a single GPS point for each vine and attempting to programmatically 


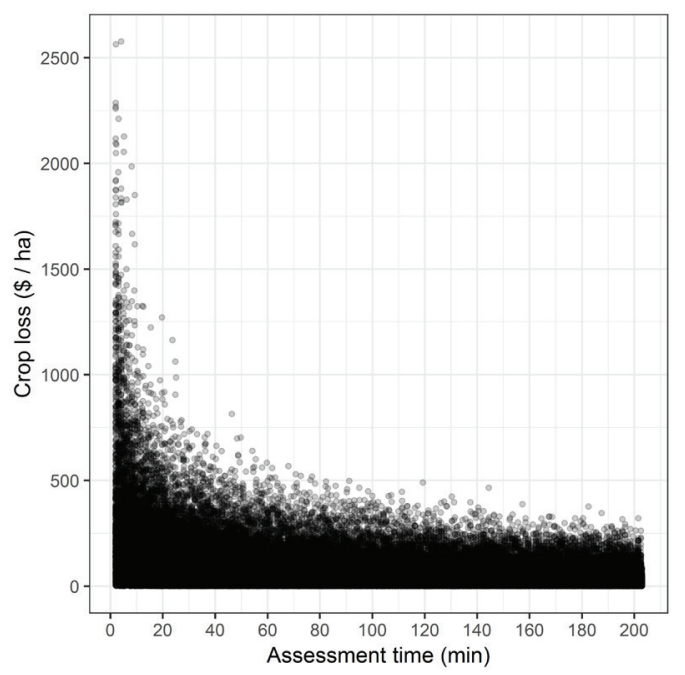

Figure 4 Effect of assessment time on potential additional crop loss (NZ\$ per hectare based on 2017 figures) as a result of errors in botrytis bunch rot (BBR) assessments affecting crop price. Crop loss shown is because of errors in assessments, and would be additional to crop loss from overall mean BBR. Data are combined across all three vineyard blocks.

assign bunches to that vine was problematic. A better approach may be to use a base-rover RTK GPS system that records a position every second, which would allow a single position to be assigned to each bunch, and then to aggregate those to the nearest vine. This approach could eliminate the need to assign bunches to vines altogether, as the vine does not affect the way in which BBR travels from bunch to bunch if the vine variety and clone is constant, and so is not essential in understanding the distribution of BBR. Additionally, canes of neighbouring vines often overlap, so bunches belonging to one vine may in fact be closer to the trunk of its neighbour.

The number of vines assessed was a function of the time available to do the assessments and the layout of the chosen block. One advantage of this inconsistency is that interpolation methods for both vines and BBR, as well as travel times and costs, could be tested on different block shapes. For example, the poorly correlated interpolated BBR values at GimblettRd1 were likely because of the long, thin block shape and the fact that the distances between assessed vines was greater. Likewise, the minimum sampling time effect seen with driving was most likely because of having to travel up and down every row once when sampled bunches were located at both ends of all rows, and this effect was more pronounced in the longer GimblettRd1 block than the squarer KorokipoRd block. Real-world vineyard blocks are seldom regular shapes, so testing sampling methodologies on irregular shapes provides more realistic results. Using precise vine positions as opposed to a rasterised vineyard grid makes this sort of irregular spatial analysis much more feasible.

Sampling times were almost completely dominated by $\mathrm{BBR}$ assessments rather than travel time, as sample sizes of more than two vines when driving or 11 vines when walking meant BBR assessments took longer than the maximum travelling time. This is based on an assumption that BBR assessments take $1 \mathrm{~min}$, but it is unlikely the assessments would be much quicker. If it was not possible to walk between rows because of vines being too difficult to duck under, then travel time may become a factor in overall assessment time. These figures are also for relatively small vineyard blocks. In reality, the distances would be greater and fatigue would become a factor if walking, necessitating the need to drive.

The economic analyses of the potential additional crop losses because of errors in BBR assessments were very simplistic and assumed that the price paid for the crop is the total potential value of the crop minus the estimated percentage of BBR. In reality, it is likely to be a lot more complicated than this and a more indepth economic analysis of disease sampling, including such items as fuel costs and ongoing disease management requirements, would make for a useful study. However, what this analysis shows is that inappropriate sample sizes have the potential for substantial economic effects, and that spending a relatively short time performing a 
reasonable assessment could result in noticeable financial savings.

\section{CONCLUSIONS}

Spatial interpolation was a successful means of studying BBR sampling strategies in vineyard blocks without needing to sample every vine. The accuracy of that interpolation depended on block shape and distance between assessed vines which needs to be accounted for in future studies.

Travel times were not found to be substantial when either driving or walking, so distance between samples should not play a factor in determining a sampling strategy.

Sample size played the biggest role in assessment times, with sample sizes of two vines when driving or 11 vines when walking taking longer to assess than the maximum travelling time. However, even when assessing enough vines to reach a point where further vines did not substantially reduce variation ( $\sim 60$ vines), the total assessment time was $80 \mathrm{~min}$ (assuming 1 min per vine assessment). This does not seem like a substantial amount of time to spend, potentially once a season, considering the economic benefits that could result from more accurate assessments.

\section{ACKNOWLEDGEMENTS}

We would like to thank the growers who allowed us to collect data on their vineyards, and Jian Liu and Matthew Norris (Plant \& Food Research) for help with operating the GPS equipment. This research was supported by The New Zealand Institute for Plant and Food Research Limited's Strategic Science Investment Fund (SSIF) from the New Zealand Ministry of Business, Innovation and Employment (MBIE).

\section{REFERENCES}

Arnold K, Golino DA, McRoberts N 2016. A synoptic analysis of the temporal and spatial aspects of grapevine leafroll disease in a historic Napa vineyard and experimental vine blocks. Phytopathology 107: 418-426.

Bramley RGV 2010. Precision viticulture: managing vineyard variability for improved quality outcomes. In: Reynolds AG Ed. Managing wine quality. Oxford, Woodhead Publishing Limited. Pp. 445-480.

Bramley RGV, Evans KJ, Dunne KJ, Gobbett DL 2011a. Spatial variation in response to 'reduced input' spray programs for powdery mildew and botrytis identified through whole-of-block experimentation. Australian Journal of Grape and Wine Research 17: 341-350.

Bramley RGV, Trought MCT, Praat J-P 2011b. Vineyard variability in Marlborough, New Zealand: characterising variation in vineyard performance and options for the implementation of Precision Viticulture. Australian Journal of Grape and Wine Research 17: 72-78.

Cochran WG 1977. Sampling Techniques. 3rd ed, John Wiley \& Sons, New York, NY, USA.

Franke R 1979. A critical comparison of some methods for interpolation of scattered data. Rep. NPS-53-79-003. Department of Mathematics, Naval Postgraduate School, Monterey, CA, USA. Retrieved 21 March 2019 from http://hdl.handle.net/10945/35052.

Gebhardt A, Bivand R, Sinclair D 2018. interp: Interpolation methods. $\mathrm{R}$ package version 1.0-31.

Geiger CA, Daane KM 2001. Seasonal movement and distribution of the grape mealybug (Homoptera: Pseudococcidae): Developing a sampling program for San Joaquin Valley vineyards. Journal of Economic Entomology 94: 291-301.

Gentle JE, Härdle WK, Mori Y 2012. Handbook of computational statistics: concepts and methods. Springer-Verlag Berlin Heidelberg. $1192 \mathrm{p}$.

González-Fernández AB, Rodríguez-Pérez JR, Sanz-Ablanedo E, Valenciano JB, Marcelo V 2019. Delineating vineyard zones by fuzzy $\mathrm{K}$-means algorithm based on grape sampling variables. Scientia Horticulturae 243: 559566.

Hahsler M, Hornik K 2018. TSP: Traveling salesperson problem (TSP). $\mathrm{R}$ package version 1.1-6. 
Hijmans RJ 2017. geosphere: Spherical trigonometry. R package version 1.5-7.

Hill GN, Beresford RM, Evans KJ 2010. Tools for accurate assessment of botrytis bunch rot (Botrytis cinerea) on wine grapes. New Zealand Plant Protection 63: 174-181.

Hill GN, Evans KJ, Beresford RM, Dambergs RG 2014. Comparison of methods for the quantification of botrytis bunch rot in white wine grapes. Australian Journal of Grape and Wine Research 20: 432-441.

Hill GN, Henshall WR, Beresford RM 2017. Manipulating rainfall to study symptom expression of Botrytis cinerea infection in wine grapes. New Zealand Plant Protection 70: 301-309.

Ifoulis A, Savopoulou-Soultani M 2006. Developing optimum sample size and multistage sampling plans for Lobesia botrana (Lepidoptera: Tortricidae) larval infestation and injury in northern Greece. Journal of Economic Entomology 99: 1890-1898.

Kahle D, Wickham H 2013. ggmap: Spatial Visualization with ggplot2. The R Journal 5: 144-161.

Kasimatis AN, Vilas EP 1985. Sampling for degrees brix in vineyard plots. American Journal of Enology and Viticulture 36: 207213.

McDonald T 2016. SDraw: Spatially balanced sample draws for spatial objects. R package version 2.1.3.

McKay AH, Hill GN, Beresford RM 2012. Comparison of sampling procedures for estimating botrytis bunch rot incidence in New Zealand vineyards. New Zealand Plant Protection 65: 241-248.

Meyers JM, Vanden Heuvel JE 2014. Use of Normalized Difference Vegetation Index images to optimize vineyard sampling protocols. American Journal of Enology and Viticulture 65: 250.

Mgocheki N, Addison P 2009. Incorporating sampling precision into an action threshold for monitoring ant (Hymenoptera: Formicidae) population levels in vineyards. Crop Protection 28: 257-263.
New Zealand Winegrowers 2018. Annual Report 2018. Retrieved 21 March 2019 from https:// www.nzwine.com/media/9567/nzw-annualreport-2018.pdf.

Park Y-L, Perring TM, Krell RK, Farrar CA, Gispert C 2006. Spatial distribution of Pierce's disease in the Coachella Valley: Implications for sampling. American Journal of Enology and Viticulture 57: 220-225.

Park Y-L, Perring TM, Krell RK, Hashim-Buckey JM, Hill BL 2011. Spatial distribution of Pierce's disease related to incidence, vineyard characteristics, and surrounding land uses. American Journal of Enology and Viticulture 62: 229-238.

Pebesma EJ, Bivand RS 2005. Classes and methods for spatial data in R. R News 5: 9-13.

RCore Team 2018. R: Alanguage and environment for statistical computing, R Foundation for Statistical Computing, Vienna, Austria.

Reynolds AG, Cliff M, Wardle DA, King M 2004. Evaluation of winegrapes in British Columbia: 'Chardonnay' and 'Pinot noir' clones. HortTechnology 14: 594-602.

Reynolds AG, de Savigny C 2004 Experiences with spatial variation of yield and fruit composition in premium vinifera vineyards in Niagara. 33rd Annual New York Wine Workshop, 31 March - 02 April 2004, Geneva, NY, USA.

Robertson BL, Brown JA, McDonald T, Jaksons P 2013. BAS: Balanced acceptance sampling of natural resources. Biometrics 69: 776-784.

Sathya N, Muthukumaravel A 2015. A review of the optimization algorithms on traveling salesman problem.

Seem R, Magarey P, McCloud P, Wachtel M 1985. A sampling procedure to detect grapevine downy mildew. Phytopathology 75: 12521257.

Taylor JA, Bates TR2012. Sampling and estimating average pruning weights in concord grapes. American Journal of Enology and Viticulture 63: 559-563.

Trought MCT, Bramley RGV 2011. Vineyard variability in Marlborough, New Zealand: characterising spatial and temporal changes 
in fruit composition and juice quality in the vineyard. Australian Journal of Grape and Wine Research 17: 79-89.

Wickham H 2016. ggplot2: Elegant Graphics for Data Analysis, Springer-Verlag New York.

Wickham H 2017. tidyverse: Easily install and load 'Tidyverse' packages. $\mathrm{R}$ package version 1.2.1. 